\title{
La sociedad civil como núcleo problemático de la teoría democrática
}

Cuando celebramos la aparición de un buen libro siempre experimentamos una agradable sensación de satisfacción. Este gozo o natural alegría se incrementa notablemente cuando el libro en cuestión es, además un libro destinado, por sus indudables virtudes, a renovar las perspectivas de discusión y comprensión de uno de los debates más complejos y debatidos de las ciencias sociales contemporáneas. En efecto, el libro presentado en estas líneas, La sociedad civil. De la teoría a la práctica, tal y como su título promete, posee el destacado mérito de ofrecer una valiosá, inteligente y crítica presentación del debate en torno a la importancia teórica y práctica de la sociedad civil entendida como nuevo paradigma de interpretación de la acción colectiva y como lugar de conjunción de las complejas áreas de definición de la problemática cuestión democrática. En particular, para el contexto mexicano, este estupendo libro coordinado por Alberto J. Olvera, cumple asertivamente con la misión de llenar un injustificable vacío en el correcto planteamiento de las muchas cuestiones nucleares para el discurso democrático presentes en la discusión de la naturaleza, el papel y el verdadero potencial emancipador de la sociedad civil en México.

Esta empresa, ciertamente, posee dificultades intrínsecas que no pueden ocultarse apelando al socorrido dato que reafirma y destaca el relevante papel democratizador desempeñado por la sociedad civil a lo largo de los procesos de transición de regímenes autoritarios a regímenes democráticos. De hecho, ese exitoso carácter de referente universal que la sociedad civil ha adquirido ante la pluralidad disímbola de los actores sociales más significativos adquiere sentido y únicamente puede sostenerse en razón de su inmanente y compleja vaguedad conceptual. Justamente, ha sido dicha vaguedad conceptual la que ha servido de frágil 
soporte al presuntamente universal valor estratégico asignado al "viejo y polémico concepto de sociedad civil" para inspirar y justificar "la formación de oposiciones políticas fuertes democráticamente organizadas". Es decir, sin pretender demeritar ese destacado papel desempeñado por la sociedad civil en diversos procesos de emancipación política y ampliación democrática a lo largo del orbe, lo cierto es que su correcta utilización para lograr una mejor interpretación empírica de procesos político concretos o para alcanzar nuevas perspectivas normativas conceptuales presupone una delimitación teórica rigurosa de los términos constitutivos de esta noción. De hecho, la principal contribución del conjunto de ensayos aquí reseñados indican cuales son aquellos elementos que forman parte de esa necesaria y positiva problematización del concepto de sociedad civil.

Ahora bien, para entrar directamente en materia, cabe destacar las principales contribuciones presentes en el conjunto de artículos que configurar al presente texto. En primer lugar, encontramos un esbozo claro y útil de los contornos esenciales que caracterizan a la discusión sobre la sociedad civil hoy. En segundo lugar, nos ofrece un recuento básico de los puntos problemáticos frente a los que se debe tomar posición para sortear los principales equívocos y obstáculos presentes emanados de la "confusión reinante" respecto de esta noción referida. Finalmente, el texto ofrece un grupo de relevantes aportes teóricos y prácticos para transformar el abusado eslogan de la sociedad civil en una perspectiva heurística poderosa y necesaria para aclarar las posibilidades y los límites de los procesos de democratización en curso en muchas sociedades contemporáneas entre las que se debe incluir, obviamente, a México.

Sobre los contornos de la discusión y el uso correcto de la noción de sociedad civil algunas contribuciones que integran el presente texto destacan un grupo de problemáticas tesis constitutivas que, al menos en México, no han sido tomadas en cuenta con la seriedad y profundidad exigida a la hora de la utilización del referido concepto. Debido a la necesaria brevedad espacial que el formato de una reseña impone quisiera referirme en concreto a dos aspectos sustantivos en este plano de la acotación del concepto.

En primer lugar, debe destacarse la reafirmación de la polisemia y ambigüedad conceptual que afectan al referente de sociedad civil (y sus equivalentes sustitutos utilizados con mayor frecuencia como son las nociones de organizaciones civiles o tercer sector) la transforman en una categoría excesivamente amplia y generalizada que desafortunadamente permite utilizarla en el plano teórico para decir con ella todo y nada a la vez. En realidad, la sociedad civil es una noción sumamente compleja y la historia misma de su desarrollo conceptual exhibe dimensiones 
laberínticas que, para tratar de decirlo en pocas palabras, muestra cómo ella ha sido alimentada por diversas tradiciones conceptuales, como lo son el liberalismo, el republicanismo y el comunitarismo — sólo para citar las fuentes o marcos normativos más relevantes en la alimentación filosófica de los grandes debates conceptuales en la teoría política contemporánea. Naturalmente, este dato se vuelve más problemático cuando nos permite comprender como la mezcla de estos principios normativos emanados de tradiciones diversas e incluso antagónicas entre sí permiten más de una interpretación a la comprensión o lectura empírica de los fenómenos asociados con el concepto de sociedad civil. Se trata, por lo demás, de fenómenos de innegable importancia y actualidad que, como sabemos, afirman de maneras diversas y con fuerza creciente la autonomía específica de lo social respecto de la economía y la política.

El segundo aspecto directamente vinculado con la complejidad de la noción de sociedad civil posee una clara orientación histórica. Como sabemos, la sociedad civil surge como manifestación de un largo despliegue del proceso de construcción y desarrollo de la modernidad en occidente. Ello, en efecto, implica que, como tendencia histórica su pleno desenvolvimiento únicamente se alcanza (e, incluso aquí de manera desigual) en las naciones emblemáticas de la modernidad. Esto nos coloca, sin duda, frente a un problema de difícil solución. La importación del concepto (muchas veces acrítica) reviste de nuevas complicaciones a la hora de explicar los procesos de aparición y permanencia de nuevos y diversos actores y movimientos sociales en sistemas políticos como los latinoamericanos aún aquejados de claros rasgos premodernos. De hecho, se vale recordar que se considera que una sociedad civil es fuerte y consolidada cuando su dinamismo particular da paso a formas de vida asociativa sostenidas en su vitalidad sobre la base del ejercicio de una efectiva ciudadanía la cual a su vez exige, para ser creíble, la presencia concomitante de, por una parte, un genuino estado de derecho y, por la otra, la existencia de una conciencia y/o cultura política proclive a estimular las prácticas democráticas genuinas. No es este el caso, lamentablemente, de la mayoría -si es que acaso no de todas- las naciones latinoamericanas en donde su desigual y forzado proceso modernizador solamente de manera muy tardía ha permitido el incipiente surgimiento y un relativo desarrollo de una sociedad civil autónoma apoyada en una esfera pública en construcción y en un conjunto de derechos ciudadanos aún no plenamente vigentes ni estables.

Este amplio, impreciso y problemático contorno de la sociedad civil indica claramente algunas de las principales dificultades teóricas y prácticas que hay que remontar para recuperar de manera rigurosa y ventajosa tal categoría. 
En efecto, resulta urgente superar cierta concepción ampliamente vulgarizada que pretende o desea fijar a la sociedad civil en (a) un espacio físico predeterminado, (b) con un actor específico que supuestamente la representa y, finalmente (c) con un único principio organizador e interpretativo de su contenido. Estos tres falsos presupuestos, ciertamente, contaminan de manera inequívoca un apropiado acercamiento a la comprensión normativa y empírica del fenómeno en cuestión volviendo más difícil la ya de por sí complicada empresa de creativa reconstrucción del concepto de sociedad civil.

Así, con relación al tema que reafirma el carácter impreciso y, sobre todo, fluctuante de las fronteras que circunscriben a la sociedad civil cabe admitir que ello significa que el metafórico locus que ubica a la sociedad civil a medio camino entre las esferas de lo público (Estado) y lo privado (mercado) simplemente busca enfatizar el carácter autónomo que corresponde a los social (sociedad civil). En consecuencia, este reconocimiento de la existencia de un entorno flexible más bien reitera la dimensión dinámica, cambiante y transformadora que define a la siempre tensa relación entre los diversos subsistemas sociales.

Asociada a esta extendida idea se encuentra la segunda tendencia indicada líneas arriba que erróneamente trata de circunscribir a un determinado actor la representación de este supuesto espacio único. Son muy frecuentes, ciertamente, las declaraciones de individuos y organizaciones que se arrogan el pleno derecho de representatividad de los intereses de la sociedad civil. A fuerza de ser una actitud excluyente y en esencia antidemocrática, tal pretensión también desconoce el carácter constitutivamente plural y conflictivo de la sociedad civil cuando esta es correctamente entendida. Evidentemente, dicho carácter fragmentada e, incluso, dispersa supone un cierto grado de dificultad en el acercamiento epistemológico del concepto pero, por otra parte, este reconocimiento abre la posibilidad de dar cabida a la diversidad de intereses sociales que alimentan el dinamismo de la sociedad en su conjunto y permite comprender y ensayar (e, incluso, combinar imaginativamente) distintos principios normativos de integración social.

Para terminar de acotar la revisión de este pequeño grupo de falsos y problemáticos presupuestos, quisiera decir rápidamente que la referida necesidad conceptual de reducir la complejidad del fenómeno de la sociedad civil a un único principio explicativo ha terminado reconociendo la cara insuficiencia e inviabilidad conceptual de dicho esfuerzo. En cierto sentido, esta es una cuestión aún abierta pero no cabe duda que incluso los más serios y calificados esfuerzos por atrapar el núcleo organizativo de la sociedad civil (piénsese por ejemplo en Habermas y su propuesta 
de integración comunicativa como eje de articulación de la sociedad civil y la esfera pública) terminan por admitir las muchas cuestiones cruciales que quedan fuera de la teoría cuando se privilegia un único principio explicativo.

Si todo lo dicho anteriormente es exacto, la teoría de la sociedad civil tiene como problemática metodológica básica encontrar un punto de partida seguro desde el cual pueda establecer una objetiva relación crítica con la realidad política empírica de la que se ocupa. Por ello, la importancia de una aproximación histórico comparativa a este concepto salta inmediatamente a la vista, así como la posibilidad y necesidad de apoyarse en una definición sistémica propia del Estado y del mercado de la integración social propiciada por la comunicación entre los seres humanos". Justamente, en la sólida argumentación a favor de estos dos principios apuntados es donde radica a mi juicio la principal aportación conceptual del presente volumen.

Dicho de otra manera, dentro de las muchas y estupendas aportaciones conceptuales y empíricas que podría resaltar en el ejercicio de comentar este volumen elijo dos que me parecen fundamentales. La primera, evidentemente, tiene que ver con el alcance y los contenidos de una definición de sociedad civil que sea capaz de resolver las dificultades emanadas de la amplitud y complejidad de esta añeja y laberíntica noción conceptual. La segunda, en cambio, corresponde a aquel conjunto de obligadas consideraciones histórico culturales que deben tomarse en cuenta a la hora de utilizar correctamente este paradigma innovador en la interpretación de realidades como las latinoamericanas marcadas por, como decía, un proceso de modernización sesgado, limitado y, en tal sentido, nada propicio a la procuración de un proceso de integración política sustentado en el reconocimiento de la pluralidad y de la autonomía de lo social.

Resulta pertinente, en consecuencia, decir unas rápidas palabras con relación a la propuesta de definición del concepto de sociedad civil. Las principales aportaciones al respecto corren de la mano de Andrew Arato y Jean Cohen, a la sazón, los autores más innovadores y sugerentes que han escrito sobre este tema en los últimos años. Ellos, como sabemos, adoptando una declarada perspectiva habermasiana debidamente problematizada y corregida, proponen un concepto de sociedad civil sustentado en una estructura dual (comunicativa y de afirmación de derechos universales) que es capaz de permitir una interpretación objetiva de los complejos matices que emanan de la relación entre los distintos subsistemas sociales. De esta suerte, la sociedad civil es entendida desde su carácter de arena de intermediación en donde la vida social busca alcanzar y mantener un difícil equilibrio sustentado en las prácticas y acciones comunicativas 
de los actores sociales involucrados. Por tanto, la estructura dual de la sociedad civil refleja la relación de recíproca influencia y codeterminación establecida entre la esfera pública y su expresión comunicativa, por un lado, y, por el otro, la esfera institucional participativa demarcada, justamente, por el conjunto de derechos sociales y políticos creados por la modernidad.

Debe insistirse en que la aparente visión estática que emana de esta propuesta conceptual indudablemente es autocorregida sobre la base del presupuesto que considera posible la existencia de la sociedad civil únicamente como resultado de su constante movimiento interno. De hecho, los principales correctivos de Cohen y Arato a la visión habermasiana de la sociedad civil parte, precisamente, del reconocimiento a la imposibilidad de hacer abstracción de las relaciones de poder en la vida cotidiana de cualquier orden social. Por tanto, esta propuesta conceptual de los aspectos -complementarios, como decía- presentes en la interpretación simultanea de la sociedad civil como institución y como movimiento social recalca tanto la incertidumbre permanente a la que se ve sometida la reproducción de la vida autónoma de la sociedad como del carácter intrínsecamente conflictivo que esta reproducción posee.

Este enfoque conceptual plantea, sin duda, muchos problemas que complican el análisis empírico de una sociedad civil concreta que evidencia en su comportamiento un proceso desigual de desarrollo de la acción de sus propios actores sociales. Pero, sin duda, no se trata de un enfoque conceptual acrítico, autocomplaciente e incapaz de tomar en cuenta los obstáculos que debe superar un proceso comunicativo sustentado en el intercambio de ideas, principios e intereses sociales diversos. Esta es, naturalmente, una ventaja nada desdeñable a la que se suma el original esfuerzo por proponer tal definición de sociedad civil desde un consciente esfuerzo por refundarla desde una recuperación y ampliación de las principales tradiciones normativas que están a su base y apuntando hacia una mejor comprensión de aquellos procesos de "racionalización sociocultural a partir de la comunicación"

Con relación al segundo aporte destacado, el cual hace énfasis en la necesidad de una crítica aproximación histórico comparativa en el estudio concreto de sociedades civiles particulares, cabe señalar que tal afirmación representa efectivamente la mejor vía para enfrentar los muchos retos que el estudio empírico de la sociedad civil plantea a ordenamientos socioculturales con rasgos similares a los que configuran a las sociedades latinoamericanas. Entre rasgos similares a los que configuran a las sociedades latinoamericanas. Entre estos retos destacan: la necesidad de fortalecer la libertad asociativa (con todo lo que ello implica), la necesidad de 
expandir a la sociedad civil más allá de su limitado horizonte urbano, la necesidad, en una palabra, de construir a la sociedad civil desde ese proceso simultáneo de desarrollo institucional y movimiento social.

Para concluir, una última y breve reflexión. Está claro que, para profundizar y consolidar los avances en materia de democratización registrados en nuestras naciones a lo largo de las últimas dos décadas, no basta con señalar la centralidad del fenómeno de emergencia de la sociedad civil. Es inevitable dar un conjunto de pasos sucesivos en la dirección de posibilitar una mayor participación democrática ciudadana como prerrequisito para extender la democracia en las circunstancias contemporáneas. Y ello no se podrá lograr sin una previa delimitación teórica precisa de los términos constitutivos de la noción de sociedad civil y sin una fuerte dosis de imaginación y deseo democratizador. Guardo la satisfacción de que, en ambas direcciones, el presente libro ofrece avances significativos, creativos y rigurosos. 